\title{
MODULE WEAK BANACH-SAKS AND MODULE SCHUR PROPERTIES OF HILBERT $C^{*}$-MODULES
}

\author{
MICHAEL FRANK AND ALEXANDER PAVLOV
}

\begin{abstract}
Continuing the research on the Banach-Saks and Schur properties started in (cf. [10) we investigate analogous properties in the module context. As an environment serves the class of Hilbert $C^{*}$-modules. Some properties of weak module topologies on Hilbert $C^{*}$-modules are described. Natural module analogues of the classical weak Banach-Saks and Schur properties are defined and studied. A number of useful characterizations of properties of Hilbert $C^{*}$-modules is obtained. In particular, some interrelations of these properties with the self-duality property of countably generated Hilbert $C^{*}$-modules are established.
\end{abstract}

\section{INTRODUCTION}

In the present paper we extend the considerations of [14, 10] on the Banach-Saks and Schur properties from the situation of Banach spaces to the situation of certain Banach $C^{*}$-modules. For Hilbert $C^{*}$-modules we introduce some natural module analogues of the weak Banach-Saks and Schur properties and study their behavior. The main idea of these constructions is to replace the weak topology of Banach spaces by a weak topology of Hilbert $C^{*}$-modules. But there are at least two suitable candidates for such a replacement - the weak topology generated by the inner product and the weak topology generated by $A$-linear bounded functionals on the Hilbert $C^{*}$-module. We considered both of them, but mostly we are interested in the first variant. In the third paragraph we obtain some properties of the mentioned weak module topologies. The forth paragraph is dedicated to the study of the module Schur property, where we investigate such important cases as standard, countably generated and finitely generated projective modules. In the fifth paragraph we focus on the study of two variants of the weak module Banach-Saks property. In the last paragraph some interrelations between both the module Schur and the module weak Banach-Saks properties and self-duality of Hilbert $C^{*}$-modules are considered.

\section{Preliminaries}

Let us recall that a (right) pre-Hilbert $C^{*}$-module over a $C^{*}$-algebra $A$ (cf. [22]) is a right $A$-module $V$ equipped with an $A$-valued inner product $\langle\cdot, \cdot\rangle: V \times V \rightarrow A$, which is $A$-linear in the second variable, fulfils $\langle x, y\rangle=\langle y, x\rangle^{*}$; is positive, i.e. $\langle x, x\rangle \geq 0$, and is definite, what means $\langle x, x\rangle=0$ if and only if $x=0$. A pre-Hilbert A-module is a Hilbert $A$-module provided it is a Banach space with respect to the norm $\|x\|=\|\langle x, x\rangle\|^{1 / 2}$. Hilbert modules over the field of complex numbers $\mathbb{C}$ are Hilbert spaces.

Partially supported by the RFBR (grant 07-01-00046), by the joint RFBR-DFG project (RFBR grant 07-01-91555 / DFG project "K-Theory, $C^{*}$-algebras, and Index theory"), and by the 'Italian project Cofin06 - Noncommutative Geometry, Quantum Groups and Applications'. 
Let $V_{1}, V_{2}$ be Hilbert $A$-modules. Then $\operatorname{Hom}_{A}\left(V_{1}, V_{2}\right)$ stands for the set of all $A$ linear bounded operators from $V_{1}$ to $V_{2}$. When $V_{1}=V_{2}=V$ we will write $\operatorname{End}_{A}(V)$ instead of $\operatorname{Hom}_{A}(V, V)$. An operator $T \in \operatorname{Hom}_{A}\left(V_{1}, V_{2}\right)$ admits an adjoint operator $T^{*} \in$ $\operatorname{Hom}_{A}\left(V_{2}, V_{1}\right)$ if the equality

$$
\langle T x, y\rangle=\left\langle x, T^{*} y\right\rangle
$$

holds for all $x \in V_{1}, y \in V_{2}$. Let us remark that a module operator between Hilbert $C^{*}$-modules may not have an adjoint one unlike in the Hilbert space situation (cf. [19, Example 2.1.2]). In the sequel, by $\operatorname{End}_{A}^{*}(V)$ we will denote the subset of $\operatorname{End}_{A}(V)$ which consists of operators that possess an adjoint one. Both these Banach algebras can be characterized alternatively in terms of different kinds of multiplier algebras of the $C^{*}$ algebra $\mathrm{K}_{A}(V)$ of "compact" operators generated as the norm-closure of the linear hull of the set of elementary operators $\left\{\theta_{x, y}: \theta_{x, y}(z):=x\langle y, z\rangle, x, y \in V\right\}$ on $V$, cf. [28, Th. 15.2.12, 15.H].

To give some information on multiplier theory consider the canonical isometric embedding of a $C^{*}$-algebra $A$ into its bidual Banach space $A^{* *}$, which may be equivalently described as the enveloping von Neumann algebra of $A[5]$. Then (left, right) multipliers of $A$ may be defined as the $C^{*}$-algebra $M(A)=\left\{a \in A^{* *}: a A \subset A ; A a \subset A\right\}$ of multipliers of $A$, and as the Banach algebra of left (resp., right) multipliers as $L M(A)=\{a \in$ $\left.A^{* *}: a A \subset A\right\}, R M(A)=L M(A)^{*}$. More information about multipliers may be found in [15, 19, 26, 28,.

Resorting to the particular case of $C^{*}$-algebras $A$ which can be considered as a right Hilbert $A$-module over themselves with the inner product $\langle a, b\rangle=a^{*} b$ for $a, b \in A$, we would like to introduce some topologies related to different kinds of multipliers of $A$. The $\mathrm{C}^{*}$-algebra $M(A)$ of multipliers of $A$ can be identified with the set $M(A)=$ $\operatorname{End}_{A}^{*}(A)$, and the Banach algebra $L M(A)$ of left multipliers of $A$ can be alternatively defined as $L M(A)=\operatorname{End}_{A}(A)$ (cf. [12, 17]). These algebras may be understood as maximal unitalizations of $A$ in some natural sense ([15, 26, 24]), whereas the $C^{*}$-algebra $A_{1}$ with adjoint identity is a minimal unitalization of $A$.

For instance, in the commutative case, i.e. for $A=C_{0}(X)$, the $C^{*}$-algebra of vanishing at infinity continuous functions on an arbitrary locally compact Hausdorff space $X$, one has $M(A)=L M(A)=C(\beta X)$ and $A_{1}=C\left(X_{+}\right)$, where $\beta X$ and $X_{+}$are the Stone-Cech and the one-point compactifications of $X$, respectively.

Keeping in mind that $A$ can be considered as a $C^{*}$-subalgebra of $\operatorname{End}_{A}^{*}(A)$, we define the strict topology on $A$ (or even on $\operatorname{End}_{A}^{*}(A)$, if it is desirable) as a locally convex topology induced by the family of semi-norms $\left\{\|\cdot\|_{a, l}:=\|\cdot a\|,\|\cdot\|_{a, r}:=\|a \cdot\|: a \in A\right\}$. Analogously, the left strict topology on $A$ (or even on $\operatorname{End}_{A}(A)$ ) is a locally-convex topology induced by the family of semi-norms $\left\{\|\cdot\|_{a, l}:=\|\cdot a\|: a \in A\right\}$. Analogously, introduce the right strict topology on $A$ as a locally convex topology induced by the family of semi-norms $\left\{\|\cdot\|_{a, r}:=\|a \cdot\|: a \in A\right\}$.

The main property of these topologies is that the completion of $A$ with respect to the strict (resp., left strict, right strict) topology in $A^{* *}$ gives the multiplier algebra $M(A)$ (resp., the left multiplier algebra $L M(A)$, the right multiplier algebra $R M(A)$ ), ([28, 9, 26]). The dual character of $A^{* *}$ as the universal $*$-representation of $A$ and as the enveloping von Neumann algebra of $A$ makes sure, that the fact remains true for 
any faithful non-degenerate $*$-representation of $A$ in another $C^{*}$-algebra of operators in certain Hilbert $C^{*}$-module replacing $A^{* *}$ as an environment for completion (cf. [9]).

For the convenience of the reader we also give a sketch of the dual module theory that will be essential in the sequel. Suppose $V$ stands for a Hilbert $A$-module again. Let $V^{\prime}=\operatorname{Hom}_{A}(V, A)$ be the set of all $A$-linear bounded maps from $V$ to $A$, the $d u a l$ (left Banach) module for $V$. Apparently there is an isometric module embedding

$$
\wedge: V \rightarrow V^{\prime}, \quad x^{\wedge}(\cdot)=\langle x, \cdot\rangle .
$$

A Hilbert $A$-module is said to be self-dual if this map is surjective. Generally speaking, $V^{\prime}$ is far not always a Hilbert $C^{*}$-module provided $A$ is just an arbitrary $C^{*}$-algebra. The inner product can be extended from $V$ to $V^{\prime}$ (and even in such way that the dual module will be self-dual) if and only if $A$ is monotone complete (cf. [7, Theorem 4.7], [11, 17], although in the more important case of $W^{*}$-algebras such an extension was described in [22]). As usual, the bidual module $V^{\prime \prime}$ should be define by the second iteration, i.e. $V^{\prime \prime}=\operatorname{Hom}_{A}\left(V^{\prime}, A\right)$. For a functional $F \in V^{\prime \prime}$ one can define a functional $\widetilde{F} \in V^{\prime}$ by the formula

$$
\widetilde{F}(x)=F(\widehat{x}), \quad x \in V .
$$

Then the map $F \mapsto \widetilde{F}$ is an $A$-module isometry from $V^{\prime \prime}$ to $V^{\prime}$, and it allows to extend the inner product from $V$ to $V^{\prime \prime}$ in the following way

$$
\langle F, G\rangle=F(\widetilde{G}), \quad F, G \in V^{\prime \prime}
$$

where the norm arising from this inner product coincides with the operator norm of $V^{\prime \prime}$. Now let $x \in V, f \in V^{\prime}$ and put

$$
\dot{x}(f)=f(x)^{*} .
$$

Then the map (2) is an isometric module embedding of $V$ in $V^{\prime \prime}$ (see [19, 23] for details). It is interesting to observe that in opposition to the situation of Banach spaces the third dual $V^{\prime \prime \prime}$ for $V$ is isomorphic to $V^{\prime}$, and the forth dual $V^{\prime \prime \prime \prime}$ is isomorphic to $V^{\prime \prime}$, whereas the Banach modules $V, V^{\prime}$ and $V^{\prime \prime}$ may be pairwise non-isomorphic in particular situations 23 .

The standard Hilbert module over a $C^{*}$-algebra $A$, that is denoted by $l_{2}(A)$ or $H_{A}$, consists of all sequences $\left(a_{i}\right)$ with elements of $A$ such that the series $\sum_{i=1}^{\infty} a_{i}^{*} a_{i}$ converge in norm. A Hilbert $C^{*}$-module $V$ is countably generated if there exists a sequence of elements of $V$ such that the set of all finite $A$-linear combinations of them is norm-dense in $V$. It is said to be finitely generated if for a finite subset of $V$ any element of $V$ can be expressed as a finite $A$-linear combination of these generators. Any finitely generated Hilbert $C^{*}$ module can be isometrically embedded into a Hilbert $A$-module of type $A^{n}$ for some finite $n \in \mathbb{N},[20$. Furthermore, Kasparov's stabilization theorem asserts that any countably generated Hilbert $A$-module can be represented as a direct orthogonal summand of the standard module $l_{2}(A)$, [12]. We need the following facts about standard modules.

Proposition 2.1. ([6], [19, Proposition 2.5.5]) Consider the set of all sequences $f=\left(f_{i}\right)$, $f_{i} \in A, i \in \mathbb{N}$, such that the partial sums of the series $\sum f_{i}^{*} f_{i}$ are uniformly bounded. If 
$A$ is a unital $C^{*}$-algebra, then this set coincides with $H_{A}^{\prime}$, where the action of $f$ on $H_{A}$ is defined by the formula

$$
f(x)=\sum_{i=1}^{\infty} f_{i}^{*} x_{i},
$$

where $x=\left(x_{i}\right) \in H_{A}$, and the norm of the functional $f$ satisfies

$$
\|f\|^{2}=\sup _{N}\left\|\sum_{i=1}^{\infty} f_{i}^{*} f_{i}\right\| .
$$

Proposition 2.2 ([6, 19]). Let $A$ be a $C^{*}$-algebra. Then the following conditions are equivalent:

(i) The Hilbert $C^{*}$-module $H_{A}$ is self-dual;

(ii) The $C^{*}$-algebra $A$ is finite-dimensional.

More information about Hilbert $C^{*}$-modules can be found in [15, 18, 19, 27, 28], for example.

\section{Weak module topologies on Hilbert $C^{*}$-Modules}

We are going to establish some properties of the topologies $\tau_{\widehat{V}}$ and $\tau_{V^{\prime}}$ for arbitrary Hilbert $C^{*}$-modules which are defined in the sequel. In particular, we want to demonstrate that whenever both these topologies coincide on a certain countably generated Hilbert module over a unital $C^{*}$-algebra then this module has to be self-dual.

Definition 3.1. Let $(V,\langle\cdot, \cdot\rangle)$ be a pre-Hilbert module over a $C^{*}$-algebra $A$, and let $V^{\prime}$ be its dual module. The $V^{\prime}$-weak module topology $\tau_{V^{\prime}}$ on $V$ is generated by the family of semi-norms

$$
\left\{\nu_{f}\right\}_{f \in V^{\prime}}, \quad \text { where } \quad \nu_{f}(x)=\|f(x)\|, x \in V,
$$

and the $\widehat{V}$-weak module topology $\tau_{\widehat{V}}$ on $V$ is generated by the family of semi-norms

$$
\left\{\mu_{z}\right\}_{z \in V}, \quad \text { where } \quad \mu_{z}(x)=\|\langle z, x\rangle\|, x \in V .
$$

Obviously, $\tau_{\widehat{V}}$ is not stronger than $\tau_{V^{\prime}}$, and these topologies coincide whenever $V$ is self-dual. What about the opposite interrelation? Do they always coincide? The next example demonstrates that for arbitrary $V$ the topologies $\tau_{\widehat{V}}$ and $\tau_{V^{\prime}}$ are distinct, in general.

Example 3.2. Let $(X, \rho)$ be a locally compact metric space, $A=C_{0}(X)$ be the corresponding $C^{*}$-algebra and $V=A$ be the Hilbert $A$-module under consideration. Suppose $\widetilde{X}=X \cup\{\widetilde{x}\}$ stands for the one-point compactification of $X$. By the commutativity of $A$ the multiplier algebras $M(A)$ and $L M(A)$ coincide. Moreover, the $A$-dual Banach $A$-module $V^{\prime}$ of $V$ can be identified with the Banach space $\operatorname{End}_{A}(A)$ which is isometrically isomorphic to the left multiplier algebra $L M(A)$ of $A$. So $V^{\prime}$ exactly coincides with the algebra $C_{b}(X)$ of all bounded continuous functions on $X$, and $V^{\prime}=C_{b}(X)$ can be considered to act by left multiplication on $V=C_{0}(X)$ (see [21, 28]). Consider a sequence 
$\left\{x_{i}\right\}$ in $X$ which converges to $\widetilde{x}$ with respect to the topology of $\widetilde{X}$. For this sequence choose a sequence of open neighborhoods

$$
O_{\delta_{i}}\left(x_{i}\right)=\left\{y \in X: \rho\left(y, x_{i}\right)<\delta_{i}\right\},
$$

under the additional request that the sequence of positive numbers $\left\{\delta_{i}\right\}$ has to be a null sequence. Let $f_{i} \in C_{0}(X)$ be elements such that $f_{i}\left(x_{i}\right)=1$ and $f_{i}=0$ outside of $O_{\delta_{i}}\left(x_{i}\right)$. Then for any $g \in C_{0}(X)$ one has

$$
\begin{aligned}
\lim _{i \rightarrow \infty}\left\|g f_{i}\right\| & =\lim _{i \rightarrow \infty} \sup _{x \in X}\left|g(x) f_{i}(x)\right|=\lim _{i \rightarrow \infty} \sup _{x \in O_{\delta_{i}}\left(x_{i}\right)}\left|g(x) f_{i}(x)\right| \\
& \leq \lim _{i \rightarrow \infty} \sup _{x \in O_{\delta_{i}}\left(x_{i}\right)}|g(x)|=0
\end{aligned}
$$

what means $\left\{f_{i}\right\}$ is a null sequence with respect to the $\tau_{\widehat{V}}$-topology. On the other hand consider the constant function $h$ on $X$, which always equals to 1 . Then $h$ belongs to $C_{b}(X)=V^{\prime}$ and

$$
\left\|h f_{i}\right\|=\sup _{x \in X}\left|f_{i}(x)\right|=1
$$

for any $i \in \mathbb{N}$. Consequently, $\left\{f_{i}\right\}$ does not converge to zero with respect to the topology $\tau_{V^{\prime}}$.

Proposition 3.3. Let $\left\{x_{n}\right\}$ be a $\tau_{\widehat{V}}$-converging sequence of a Hilbert $C^{*}$-module $V$. Then the set $\left\{x_{n}\right\}$ is bounded in norm.

Proof. Consider the sets

$$
A_{k, n}=\left\{y \in V:\left\|\left\langle y, x_{n}\right\rangle\right\| \leq k\right\}
$$

and $A_{k}=\cap_{n} A_{k, n}$. These sets are closed in norm, because the function $y \mapsto\left\|\left\langle y, x_{n}\right\rangle\right\|$ is continuous for any fixed $x_{n}$. The $\tau_{\widehat{V}}$-convergence of $\left\{x_{n}\right\}$ implies that $V=\cup_{k} A_{k}$. By the Baire's theorem there are a number $k_{0}$ and a ball $B(y, \varepsilon)=\{z \in V:\|y-z\|<\varepsilon\}$ such that $B(y, \varepsilon) \subset A_{k_{0}}$. Thus the sequence $\left\{x_{n}\right\}$, understood as a subset of $V^{\prime}$, is bounded on the ball $B(y, \varepsilon)$ and, consequently, on any ball of $V$, in particular on $B(0,1)$, that exactly means

$$
\begin{aligned}
C & \geq \sup _{\|y\| \leq 1}\left\|\left\langle y, x_{n}\right\rangle\right\|=\sup _{\|y\| \leq 1}\left\|\widehat{y}\left(x_{n}\right)\right\| \\
& =\sup _{\|y\| \leq 1}\left\|\dot{x}_{n}(\widehat{y})\right\|=\sup _{\|\widehat{y}\| \leq 1}\left\|\dot{x}_{n}(\widehat{y})\right\| \\
& =\left\|\dot{x}_{n}\right\|=\left\|x_{n}\right\|
\end{aligned}
$$

for some constant $C$ and for all $n \in \mathbb{N}$, where the properties of the maps (11) and (2) have been applied.

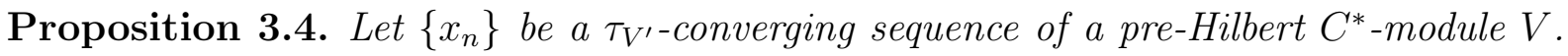
Then the set $\left\{x_{n}\right\}$ is bounded in norm.

Proof. We should only remark that $V^{\prime}$ is a Banach module even if $V$ is just a pre-Hilbert one. Further arguments are close to those applied in the proof of Proposition 3.3.

Let us remind that a subset $M$ of a topological vector space $X$ is said to be bounded if for any open neighborhood $U$ of zero in $X$ there is real number $s>0$ such that $M \subset t U$ for any $t>s$. 
Proposition 3.5. The following conditions for a subset $Q$ of a Hilbert $C^{*}$-module $V$ are equivalent:

(i) $Q$ is bounded with respect to the topology $\tau_{\widehat{V}}$;

(ii) $Q$ is norm-bounded.

Proof. Because the norm topology is stronger than the topology $\tau_{\widehat{V}}$ one has only to check the implication (i) $\rightarrow$ (ii).

Let $Q$ be bounded with respect to the topology $\tau_{\widehat{V}}$. Assume there is a sequence $\left\{x_{n}\right\} \in Q$ for which the sequence $\left\{\left\|x_{n}\right\|\right\}$ is unbounded. Because the set $\left\{x_{n}\right\}$ is $\tau_{\widehat{V}}$-bounded, for any $y \in V$ one can find a real number $t>0$ satisfying

$$
\left\{x_{n}\right\} \subset t\{x:\|\langle y, x\rangle\|<1\}
$$

what yields

$$
\left\|\left\langle y, x_{n}\right\rangle\right\|<t \text { for all } n \in \mathbb{N} .
$$

In particular, the sets $\left\{\left\|\left\langle y, x_{n}\right\rangle\right\|\right\}_{n=1}^{\infty}$ are bounded for any fixed $y \in V$. The remainder of the proof can be done in the same way like the proof of Proposition 3.3. We arrive at a contradiction since the sequence $\left\{\left\|x_{n}\right\|\right\}$ turns out to be bounded.

Proposition 3.6. The following conditions for a subset $Q$ of a pre-Hilbert $C^{*}$-module $V$ are equivalent:

(i) $Q$ is bounded with respect to the topology $\tau_{V^{\prime}}$;

(ii) $Q$ is norm-bounded.

Proof. The proof follows the arguments of the proofs of Propositions 3.3 and [3.5] with obvious changes in details.

Theorem 3.7. Let $A$ be a unital $C^{*}$-algebra, $V=l_{2}(A)$ be the standard countably generated Hilbert A-module. Suppose, the topologies $\tau_{\widehat{V}}$ and $\tau_{V^{\prime}}$ coincide on $V$. Then $V$ is self-dual and $A$ has to be finite-dimensional.

Proof. Let us take into consideration any functional $\beta \in V^{\prime}$. Then by Proposition 2.2 there are elements $b_{i}$ from $A$ such that $\beta(x)=\sum_{i=1}^{\infty} b_{i}^{*} a_{i}$, where $x=\left(a_{i}\right) \in V$ and

$$
\left\|\sum_{i=1}^{N} b_{i}^{*} b_{i}\right\| \leq C
$$

for some constant $C$ and for all $N$. By the suppositions of the theorem there are vectors $y_{1}, \ldots, y_{n}$ of $V$ and a constant $K>0$ such that

$$
\nu_{\beta}(x) \leq K \max \left\{\mu_{y_{1}}(x), \ldots, \mu_{y_{n}}(x)\right\}
$$

for any $x \in V$, where we have used the notations (3) and (4). Let us fix the notations $y_{i}=\left(y_{j}^{(i)}\right), x=\left(a_{i}\right)$, where $y_{j}^{(i)}, a_{i} \in A$ and rewrite the inequality (15) in the form

$$
\left\|\sum_{i=1}^{\infty} b_{i}^{*} a_{i}\right\| \leq K \max \left\{\left\|\sum_{j=1}^{\infty}\left(y_{j}^{(1)}\right)^{*} a_{j}\right\|, \ldots,\left\|\sum_{j=1}^{\infty}\left(y_{j}^{(n)}\right)^{*} a_{j}\right\|\right\} .
$$


For arbitrary positive integers $k$ and $N$ set $a_{i}=b_{i}$ if $k \leq i \leq k+N$, and $a_{i}=0$ otherwise. Then the formula (6) gives

$$
\begin{aligned}
\left\|\sum_{i=k}^{k+N} b_{i}^{*} b_{i}\right\| \leq K \max & \left\{\left\|\sum_{j=k}^{k+N}\left(y_{j}^{(1)}\right)^{*} b_{j}\right\|, \ldots,\left\|\sum_{j=k}^{k+N}\left(y_{j}^{(n)}\right)^{*} b_{j}\right\|\right\} \\
\leq K \max & \left\{\left\|\sum_{j=k}^{k+N}\left(y_{j}^{(1)}\right)^{*} y_{j}^{(1)}\right\|^{1 / 2}\left\|\sum_{j=k}^{k+N} b_{j}^{*} b_{j}\right\|^{1 / 2}, \ldots,\right. \\
& \left.\left\|\sum_{j=k}^{k+N}\left(y_{j}^{(n)}\right)^{*} y_{j}^{(n)}\right\|^{1 / 2}\left\|\sum_{j=k}^{k+N} b_{j}^{*} b_{j}\right\|^{1 / 2}\right\} \\
\leq C^{\frac{1}{2}} \max & \left\{\left\|\sum_{j=k}^{k+N}\left(y_{j}^{(1)}\right)^{*} y_{j}^{(1)}\right\|^{1 / 2}, \ldots,\left\|\sum_{j=k}^{k+N}\left(y_{j}^{(n)}\right)^{*} y_{j}^{(n)}\right\|^{1 / 2}\right\} .
\end{aligned}
$$

Therefore $\left\{\sum_{i=1}^{N} b_{i}^{*} b_{i}\right\}_{N=1}^{\infty}$ is a Cauchy sequence with respect to the uniform topology what ensures the vector $\left(b_{i}\right)$ to belong to $V=l_{2}(A)$. Thus, $V$ is self-dual and, by Proposition 2.2, $A$ is finite-dimensional.

Corollary 3.8. Let $A$ be a unital $C^{*}$-algebra. Let $V$ be a countably generated Hilbert A-module, for which the topologies $\tau_{\widehat{V}}$ and $\tau_{V^{\prime}}$ coincide. Then $V$ is self-dual.

Proof. By Kasparov's stabilization theorem [12] $V$ can be isometrically represented as a direct orthogonal summand of $l_{2}(A)$, also denoted by $V$ for brevity. So the Hilbert $A$-module $l_{2}(A)$ decomposes into a direct orthogonal sum $V \oplus V^{\perp}$. This orthogonal decomposition of $l_{2}(A)$ causes an direct topological sum decomposition of the $A$-dual Banach $A$-module $l_{2}(A)^{\prime}$ into the sum of the set $\left(V^{\perp}\right)^{\prime}$ of all elements of $l_{2}(A)^{\prime}$ vanishing on $V$ and the set $V^{\prime}$ of all elements of $l_{2}(A)^{\prime}$ vanishing on $V^{\perp}$. Applying the basic idea of the previous proof again and resorting to sequences in $V \subseteq l_{2}(A)$ the standard isometric embedding of $l_{2}(A)$ into its $A$-dual $l_{2}(A)^{\prime}$ is seen to map $V$ onto $V^{\prime}$. Consequently, $V$ is self-dual.

Summing up, we have shown that for countably generated Hilbert $C^{*}$-modules over unital $C^{*}$-algebras the coincidence of both the toplogies $\tau_{\widehat{V}}$ and $\tau_{V^{\prime}}$ on the Hilbert $C^{*}$ module forces self-duality of the Hilbert $C^{*}$-module, and vice versa.

\section{The module Schur Property}

In the following section we introduce a module analogue of the Schur property of Banach spaces. Recall that a Banach space $X$ has the Schur property if every weak convergent sequence in $X$ converges in norm. Let $Y$ be a closed subspace of $X$. Then $X$ has the Schur property if and only if both $Y$ and the quotient space $X / Y$ have the same property (cf. [2, 14]).

Example 4.1. The Banach space $L^{1}[0,1]$ has the Schur property. Indeed, let $\left\{x_{i}\right\}$ be a weakly convergent sequence of $L^{1}[0,1]$. Then, in particular, for a unit function $1 \in$ $L^{\infty}[0,1]=L^{1}[0,1]^{\prime}$ the sequence $\left\{1 \cdot x_{i}\right\}$ has to converge in norm, but, in fact, this sequence coincides with $\left\{x_{i}\right\}$. 
Comparing the Banach space and the Banach module variants of the Schur properties we establish properties of $C^{*}$-algebras and of Hilbert $C^{*}$-modules with the module Schur property. For classification results with respect to the classical Schur property see [14, 10].

Definition 4.2. A Hilbert $C^{*}$-module $V$ has the module Schur property if every $\tau_{\widehat{V}^{-}}$ convergent sequence in $V$ converges in norm.

Lemma 4.3. Any $\tau_{\widehat{V}}$-convergent sequence $\left\{x_{n}\right\}$ of a Hilbert $C^{*}$-module $V$ with a subsequence $\left\{x_{n(l)}\right\}$ which converges in norm to some element $x \in V$ admits $x$ as its $\tau_{\widehat{V}}$-limit. Consequently, Hilbert $C^{*}$-modules with the module Schur property are sequentially $\tau_{\widehat{V}^{-}}$ complete.

Proof. For any $z \in V$ and any $\varepsilon>0$ there exists a number $N \in \mathbb{N}$ such that

$$
\left\|\left\langle z, x_{n}-x_{m}\right\rangle\right\|<\varepsilon
$$

for any $m, n>N$. In particular, there exists a number $L \in \mathbb{N}$ such that $n(l)>N$ for any $l>L$. So

$$
\left\|\left\langle z, x-x_{m}\right\rangle\right\|=\lim _{l \rightarrow \infty}\left\|\left\langle z, x_{n(l)}-x_{m}\right\rangle\right\| \leq \varepsilon
$$

for any $m>N$, and $x \in V$ turns out to be the $\tau_{\widehat{V}}$-limit of the sequence $\left\{x_{n}\right\}$.

Let us recall that a $C^{*}$-algebra has the Schur property if and only if it is finite dimensional (cf. [14, Lemma 3.8]). Our next goal is to prove that a $C^{*}$-algebra with a strictly positive element possesses the module Schur property if and only if it is unital. The conclusion that a unital $C^{*}$-algebra has the module Schur property is obvious. But, before investigating the converse statement we will consider a couple of examples as a motivation.

Example 4.4. Let $A=V=C_{0}(0,1]$ and let us define a function $f_{n} \in A$ in the following way: $f$ equals to zero at 0 and on the interval $\left[\frac{1}{n}, 1\right], f$ equals to 1 at the point $\frac{1}{2 n}$, and $f$ is linear on both the intervals $\left[0, \frac{1}{2 n}\right]$ and $\left[\frac{1}{2 n}, \frac{1}{n}\right]$. Obviously, the sequence $\left\{f_{n}\right\}$ converges to zero with respect to the topology $\tau_{\widehat{V}}$, but it does not converge in norm. So the $C^{*}$-algebra $C_{0}(0,1]$ does not have the module Schur property.

Example 4.5. Let $A=V=K(H)$ be the $C^{*}$-algebra of compact operators in a separable Hilbert space $H$ and $B(H)$ be the set of all bounded linear operators on $H$. Consider a sequence $\left\{a_{n}\right\} \in A$ and assume that the sequence $\left\{k a_{n}\right\}$ converges in norm for any $k \in K(H)$. Then its limit with respect to the right strict topology belongs to the closure of $K(H)$ in $B(H)$, and this closure coincides with $B(H)$ (see, for instance, [16, 24]). However, the norm limit of $\left\{a_{n}\right\}$, whenever it exists, has to belong to $K(H)$. So any sequence from $K(H)$, whose right strict limit lies outside $K(H), \tau_{\widehat{V}}$-converges, but does not converge in norm. Therefore $K(H)$ does not have the module Schur property, too.

Theorem 4.6. A $C^{*}$-algebra with a strictly positive element, in particular, a separable $C^{*}$-algebra (cf. [26, 1.4.3]), has the module Schur property if and only if it is unital. In particular, the Schur property for $C^{*}$-algebras is strictly stronger than the module Schur property. 
Proof. Suppose a non-unital $C^{*}$-algebra $A$ has a strictly positive element. That condition on $A$ is equivalent to the existence of a countable approximative unit in $A$ ([26, Proposition 3.10.5]). For such $A$ any element of its right multiplier algebra $R M(A)$ may be obtained just as a limit of a sequence of $A$ converging with respect to the right strict topology, i.e. there is not any necessity to consider nets under these assumptions (cf. [28, §2.3], [19, $\S 5.5])$. Now, to check that $A$ does not have the module Schur property we will reason similar as at Example 4.5. Let a sequence $\left\{a_{n}\right\}$ of $A$ be $\tau_{\widehat{V}}$-convergent (here we identify $V=A$ again), i.e. the sequence $\left\{b^{*} a_{n}\right\}$ converges in norm for any $b \in A$. Therefore, the $\tau_{\widehat{V}}$-limit of $\left\{a_{n}\right\}$ belongs to the right strict closure of $A$ inside of its bidual Banach space $A^{* *}$, i.e. it belongs to the right multipliers $R M(A)$ of $A$. Because the algebra $R M(A)$ is strictly larger than $A$ whenever $A$ is not unital, we can find a sequence from $A$ that converges with respect to the topology $\tau_{\widehat{V}}$ (for instance, to the identity of $R M(A)$ ), but does not converge in norm. Thus $A$ does not have the module Schur property.

Since unital $C^{*}$-algebras have the Schur property if and only if they are finite-dimensional, the module Schur property is weaker than the Schur property for $C^{*}$-algebras.

The next example demonstrates the requirement of Theorem 4.6 to a $C^{*}$-algebra to have a strictly positive element is essential.

Example 4.7. Let $H$ be a non-separable Hilbert space, $A=B(H)$ be the $C^{*}$-algebra of all linear bounded operators in $H$ and $A_{0}$ denotes the set of operators $T \in B(H)$ such that both $\operatorname{Ker}(T)^{\perp}$ and $\overline{\operatorname{Range}(T)}$ are separable (closed) subspaces of $H$. Then $A_{0}$ is an involutive subalgebra of $A$, containing all compact operators on $H$. Moreover, $A_{0}$ is closed in norm. Indeed, let a sequence $\left\{T_{n}\right\}$ of $A_{0}$ converges in norm to a certain element $T$ of $B(H)$. Consider (closed) separable subspaces

$$
H_{1}=\overline{\operatorname{span}}\left\{\cup \operatorname{Ker}\left(T_{n}\right)^{\perp}\right\}, \quad H_{2}=\overline{\operatorname{span}}\left\{\cup \overline{\operatorname{Range}\left(T_{n}\right)}\right\}
$$

of $H$, generated by the unions $\cup \operatorname{Ker}\left(T_{n}\right)^{\perp}$ and $\cup \overline{\operatorname{Range}\left(T_{n}\right)}$ respectively. Then, obviously, $H_{1}^{\perp}$ coincides with the intersection $\cap \operatorname{Ker}\left(T_{n}\right)$, what infers $H_{1}^{\perp}$ belongs to $\operatorname{Ker}(T)$. This implies that $\operatorname{Ker}(T)^{\perp}$ is a subspace of $H_{1}$ and, consequently, is separable. On the other hand, $\overline{\text { Range }(T)}$ belongs to the union $\cup \overline{\operatorname{Range}\left(T_{n}\right)}$. Therefore, both $\operatorname{Ker}(T)^{\perp}$ and $\overline{\operatorname{Range}(T)}$ are separable subspaces of $H$ and $T \in A_{0}$. Clearly, the $C^{*}$-algebra $A_{0}$ is not unital, but we claim that it is not even $\sigma$-unital. To verify this, let us suppose the opposite assertion to be true, and there is a countable approximative identity $\left\{T_{n}\right\}$ in $A_{0}$. Let $H_{1}$ be defined by (77). Then there is a non-zero vector $x \in H$ such that it is orthogonal to $H_{1}$. Let $p \in B(H)$ be the orthogonal projection onto the span of $x$. Then, actually, $p$ belongs to $A_{0}$ and the sequence $p T_{n}$ does not converge in norm to $p$. So we have a contradiction and, consequently, $A_{0}$ does not have any countable approximative identity. Nevertheless, we claim that $V=A_{0}$, considered as a Hilbert $A_{0}$-module, satisfies the module Schur property. Indeed, assume $\left\{T_{n}\right\}$ is a $\tau_{\widehat{V}}$-convergent sequence of $V$. Then both spaces $H_{1}$ and $H_{2}$, defined by (7), are separable. Let $u \in B(H)$ be a minimal partial isometry between $H_{1}$ and $H_{2}$. Then, in fact, $u$ belongs to $A_{0}$, where $\left.u\right|_{H_{1}^{\perp}}=0$ and $\left.u^{*}\right|_{H_{2}^{\perp}}=0$. Thus the sequence $\left\{u^{*} T_{n}\right\}$ converges in norm and coincides element-wise with the sequence $\left\{T_{n}\right\}$.

Proposition 4.8. Assume a Hilbert $C^{*}$-module $V$ is represented as a direct sum $V=$ $V_{1} \oplus V_{2}$. Then the following conditions are equivalent: 
(i) $V$ has the module Schur property;

(ii) Both $V_{1}$ and $V_{2}$ have the module Schur property.

Proof. Obviously, (i) implies (ii) and we have just to verify the inverse assertion. So

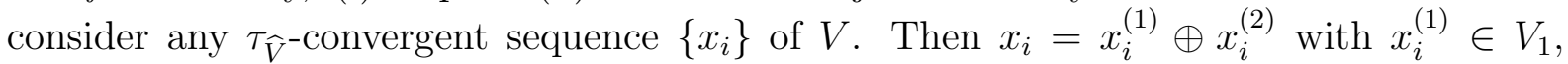
$x_{i}^{(2)} \in V_{2}$. Obviously, the sequences $\left\{x_{i}^{(j)}\right\}$ are $\tau_{\widehat{V}_{j}}$-convergent sequences $(j=1,2)$, and therefore, they converge with respect to the norm of $V_{j}(j=1,2)$. Consequently,

$$
\left\|x_{n}-x_{m}\right\| \leq\left\|x_{n}^{(1)}-x_{m}^{(1)}\right\|+\left\|x_{n}^{(2)}-x_{m}^{(2)}\right\|
$$

for any $n, m$. Thus, $\left\{x_{i}\right\}$ is a Cauchy sequence with respect to the norm topology of $V$.

Corollary 4.9. Finitely generated Hilbert modules over unital $C^{*}$-algebras $A$ have the module Schur property. In particular, the standard Hilbert $A$-modules $A^{n}$ for $n \in \mathbb{N}$ have the module Schur property.

Proof. For any Hilbert $A$-module $A^{n}$ and for any $n \in \mathbb{N}$ we can apply Proposition $4.8 n$ times, since $A$ is shown to have the module Schur property by Theorem 4.6.

By the Kasparov stabilization theorem (cf. [12], [19, Theorem 1.4.2]) any finitely generated module $V$ is an orthogonal direct summand of the standard module $l_{2}(A)$. Therefore, by [19, Theorem 2.7.5] (or by [20]) it has to be projective whenever $A$ is unital. So, $V$ is a direct summand of some Hilbert $A$-module $A^{n}, n<\infty$, and Proposition 4.8 applies in the inverse direction. So any projective finitely generated Hilbert module over a unital $C^{*}$-algebra has the module Schur property.

Corollary 4.10. Let $A$ be a $\sigma$-unital $C^{*}$-algebra with the module Schur property. Then finitely generated projective Hilbert modules over A have the module Schur property.

Proof. The assertion directly follows from Theorem 4.6 and Proposition 4.8.

Proposition 4.11. The standard Hilbert module $V=l_{2}(A)$ does not have the module Schur property for any $C^{*}$-algebra $A$.

Proof. For a unital $C^{*}$-algebra the statement is clear, because the standard basis $\left\{e_{i}\right\}$ (with all entries of $e_{i}$ equal to zero except the $i$-th, which equals $1_{A}$ ) $\tau_{\widehat{V}}$-converges to zero, but does not converge in norm.

For an arbitrary $C^{*}$-algebra $A$ we can reason in the following way: let us fix an element $a \in A$ of norm one and consider the sequence $\left\{x_{k}\right\}$ from $l_{2}(A)$, where all entries of $x_{k}$ are zero except the $k$-th entry that equals to $a$. Then for any $y=\left(b_{i}\right)$ from $l_{2}(A)$ one has

$$
\left\|\left\langle y, x_{k}\right\rangle\right\|=\left\|b_{k}^{*} a\right\| \leq\left\|b_{k}\right\|
$$

for each $k$, so $\left\{x_{k}\right\}$ converges to zero with respect to the topology $\tau_{\widehat{V}}$. On the other hand, this sequence does not converge in norm.

Let us consider one example of a countably, but not finitely generated and non-standard Hilbert $C^{*}$-module without the module Schur property.

Example 4.12. Let us consider the projection map $p: Y \rightarrow X$ from Figure 1, where $X$ is an interval, say $[-1,1]$, and $Y$ is the topological union of one interval with two copies 


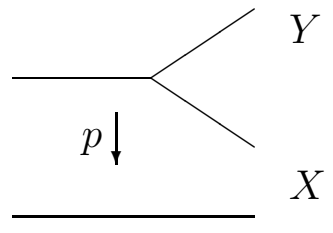

Figure 1. Example 4.12

of another half-interval with a branch point at 0 . Then $C(Y)$ is a Banach $C(X)$-module with respect to the action

$$
(f \xi)(y)=f(y) \xi(p(y)), \quad f \in C(Y), \xi \in C(X) .
$$

Let us define the $C(X)$-valued inner product on $C(Y)$ by the formula

$$
\langle f, g\rangle(x)=\frac{1}{\# p^{-1}(x)} \sum_{y \in p^{-1}(x)} \overline{f(y)} g(y),
$$

where $\# p^{-1}(x)$ is the cardinality of $p^{-1}(x)$. It was shown in 25] that $C(Y)$ is a countably, but not finitely generated Hilbert $C(X)$-module with respect to the inner product (8). Now for a point $x \in[0,1]$ let us denote by $y_{x}^{(1)}$ its pre-image $p^{-1}(x)$ intersected with the upper line of $Y$, and by $y_{x}^{(2)}$ the intersection of $p^{-1}(x)$ with the lower line of $Y$. Consider functions $h_{n} \in C(X)$ that equal zero to the left of the point $\frac{1}{2 n}$ and to the right of the point

$\frac{1}{n}$, equal 1 at the point $\frac{3}{4 n}$ and linear on both intervals $\left[\frac{1}{2 n}, \frac{3}{4 n}\right]$ and $\left[\frac{3}{4 n}, \frac{1}{n}\right]$. Now we define a sequence $\left\{f_{n}\right\}$ from $C(Y)$ in the following way: $f_{n}=0$ on $p^{-1}([-1,0]), f_{n}\left(y_{x}^{(1)}\right)=h_{n}(x)$ and $f_{n}\left(y_{x}^{(2)}\right)=-h_{n}(x)$ for all $x \in[0,1]$. Then for any $g \in C(Y)$ one has

$$
\begin{aligned}
\left\|\left\langle g, f_{n}\right\rangle\right\| & =\max _{x \in\left[\frac{1}{2 n}, \frac{1}{n}\right]} \frac{1}{2}\left|\overline{g\left(y_{x}^{(1)}\right)} f_{n}\left(y_{x}^{(1)}\right)+\overline{g\left(y_{x}^{(2)}\right)} f_{n}\left(y_{x}^{(2)}\right)\right| \\
& =\max _{x \in\left[\frac{1}{2 n}, \frac{1}{n}\right]} \frac{1}{2}\left|g\left(y_{x}^{(1)}\right)-g\left(y_{x}^{(2)}\right)\right| h_{n}(x) \\
& \leq \max _{x \in\left[\frac{1}{2 n}, \frac{1}{n}\right]} \frac{1}{2}\left|g\left(y_{x}^{(1)}\right)-g\left(y_{x}^{(2)}\right)\right|
\end{aligned}
$$

and the latter sequence converges to zero if $n$ goes to infinity. But on the other hand the sequence $\left\{f_{n}\right\}$ does not converge in norm. Thus $C(Y)$ does not have the module Schur property.

\section{Module Weak Banach-Saks properties}

The basic idea of the investigations presented in this section is a search for module analogues for the different kinds of Banach-Saks properties for Banach spaces. As most promissing we select a certain generalization of the weak Banach-Saks property.

Let us start with a short review of two Banach-Saks type properties for the classical situation. A Banach space $X$ has the Banach-Saks property if from any bounded sequence 
$\left\{x_{n}\right\}$ of $X$ there may be extracted a subsequence $\left\{x_{n(k)}\right\}$ such that

$$
\lim _{k \rightarrow \infty}\left\|\frac{1}{k} \sum_{i=1}^{k} x_{n(i)}-x\right\|=0
$$

for some element $x \in X$.

Originally, this condition was introduced and studied by S. Banach and S. Saks in [1] just for particular examples, namely they demonstrated that the Banach spaces $L^{p}([0,1])$ with $1<p<\infty$ satisfy the Banach-Saks property. Let us emphasize also that a Banach space is reflexive whenever it has the Banach-Saks property [4]. A $C^{*}$-algebra has the Banach-Saks property if and only if it is finite-dimensional [14, 3].

In Functional Analysis a weaker type of the Banach-Saks property gives very useful results for larger classes of Banach spaces. More precisely, a Banach space $X$ has the weak Banach-Saks property if for any sequence $\left\{x_{n}\right\}$ of $X$ which converges weakly to zero there may be selected a subsequence $\left\{x_{n(k)}\right\}$ such that the equality (9) is fulfilled with $x=0$. We are interested in certain module analogues of the weak Banach-Saks property in this section. To give definitions, assume $V$ to be a Hilbert $C^{*}$-module. Consider the following conditions on $V$.

(mBS1) Any $\tau_{\widehat{V}}$-null sequence $\left\{x_{n}\right\}$ of $V$ admits a subsequence $\left\{x_{n(i)}\right\}$ that satisfies the equality (9) with $x=0$.

(mBS2) Any $\tau_{\widehat{V}}$-convergent sequence $\left\{x_{n}\right\}$ of $V$ admits a subsequence $\left\{x_{n(i)}\right\}$ such that the sequence $\frac{1}{k} \sum_{i=1}^{k} x_{n(i)}$ converges in norm.

Let us emphasize that the condition (9) with $x=0$ may be rewritten for Hilbert $C^{*}$-modules in the following equivalent way

$$
\lim _{k \rightarrow \infty}\left\|\frac{1}{k^{2}} \sum_{i, j=1}^{k}\left\langle x_{n(i)}, x_{n(j)}\right\rangle\right\|=0 .
$$

Obviously, any $\tau_{\widehat{V}}$-null orthogonal sequence $\left\{x_{k}\right\}$ of norm one vectors in a Hilbert $C^{*}$ module $V$ satisfies the condition (10).

Lemma 5.1. Condition (mBS2) implies condition (mBS1), but not conversely, generally speaking.

Proof. Clearly, condition (mBS2) implies condition (mBS1) as a particular case. To see the non-equivalence of these conditions consider the $C^{*}$-algebra $A=K\left(l_{2}\right)$ of all compact operators on a separable Hilbert space $l_{2}$ as a Hilbert $C^{*}$-module over itself. Then condition (mBS1) is fulfilled, however condition (mBS2) does not hold: indeed, for any sequence of minimal pairwise orthogonal projections $\left\{p_{i}\right\}$ with their least upper bound $1_{B\left(l_{2}\right)}$, where $1_{B\left(l_{2}\right)}$ denotes the unit of the $C^{*}$-algebra $B\left(l_{2}\right)$ of all bounded linear oper-

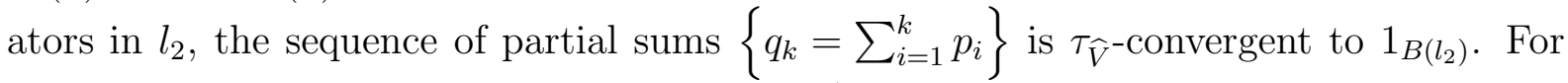
any subsequence $\left\{q_{k(j)}\right\}$ of it the sequence $\frac{1}{j} \sum_{i=1}^{j} q_{k(j)}$ converges to an infinite projection operator on $l_{2}$. So the latter sequence cannot converge in norm since infinite projection operators do not belong to $A$ which is already norm-closed. 
MODULE WEAK BANACH-SAKS AND MODULE SCHUR PROPERTIES OF HILBERT $C^{*}$-MODULE\$3

Lemma 5.2. Any $\tau_{\widehat{V}}$-convergent sequence $\left\{x_{n}\right\}$ of a Hilbert $C^{*}$-module $V$ with a subsequence $\left\{x_{n(i)}\right\}$ such that the sequence $\frac{1}{k} \sum_{i=1}^{k} x_{n(i)}$ converges in norm to some element $x \in V$ admits $x$ as its $\tau_{\widehat{V}}$-limit. Consequently, Hilbert $C^{*}$-modules with condition (mBS2) are sequentially $\tau_{\widehat{V}}$-complete.

Proof. Consider any $\tau_{\widehat{V}}$-convergent sequence $\left\{x_{n}\right\}$ of $V$ that admits a subsequence $\left\{x_{n(i)}\right\}$ such that the sequence $\frac{1}{k} \sum_{i=1}^{k} x_{n(i)}$ converges in norm to some $x \in V$. Then this sequence has to $\tau_{\widehat{V}}$-converge to the same element $x$, that means

$$
\lim _{l \rightarrow \infty}\left\|\left\langle z, \frac{1}{l} \sum_{i=1}^{l}\left(x_{n(i)}-x\right)\right\rangle\right\|=0
$$

for any $z \in V$. Therefore, for any $z \in V$, any $\varepsilon>0$ there exists an $L \in \mathbb{N}$ such that for any $k>l>L$ the inequality

$$
\varepsilon>\left\|\left\langle z, \frac{1}{l} \sum_{i=1}^{l}\left(x_{n(i)}-x\right)\right\rangle-\left\langle z, \frac{1}{k} \sum_{i=1}^{k}\left(x_{n(i)}-x\right)\right\rangle\right\|=\frac{1}{k-l}\left\|\sum_{i=l+1}^{k}\left\langle z,\left(x_{n(i)}-x\right)\right\rangle\right\|
$$

holds. Selecting $k=l+1$ we arrive at

$$
\lim _{l \rightarrow \infty}\left\|\left\langle z,\left(x_{n(l)}-x\right)\right\rangle\right\|=0
$$

and the subsequence $\left\{x_{n(l)}\right\}$ of the sequence $\left\{x_{n}\right\} \tau_{\widehat{V}}$-converges to $x \in V$. Now, for any $z \in V$ we obtain

$$
\begin{aligned}
0 & =\lim _{n \rightarrow \infty} \lim _{l \rightarrow \infty}\left\|\left\langle z,\left(x_{n}-x_{n(l)}\right)\right\rangle\right\| \\
& =\lim _{n \rightarrow \infty}\left\|\left\langle z,\left(x_{n}-x\right)\right\rangle\right\| .
\end{aligned}
$$

Consequently, the sequence $\left\{x_{n}\right\} \tau_{\widehat{V}}$-converges to $x \in V$ by definition, and this is equivalent to the assertion that the sequence $\left\{x_{n}-x\right\} \tau_{\widehat{V}}$-converges to zero.

The study of the property (mBS1) applied to the situation of Hilbert $C^{*}$-modules gives a rather surpringly general result, in comparison to the weak Banach-Saks property situation.

Theorem 5.3. Any Hilbert $C^{*}$-module has the property (mBS1).

Proof. We will reason in the vein of the original work [1]. Let $\left\{x_{k}\right\}$ be a $\tau_{\widehat{V}}$-null sequence of a Hilbert $C^{*}$-module. By Proposition 3.3 we may suppose that the norms of all the elements $x_{k}$ do not exceed one. Put $n(1)=1$. By supposition for an arbitrary $\varepsilon_{1}>0$ there exists a number $n(2)$ such that $\left\|\left\langle x_{n(1)}, x_{i}\right\rangle\right\|<\varepsilon_{1}$ whenever $i>n(2)$. Analogously for an arbitrary $\varepsilon_{2}>0$ there is a number $n(3)$ such that $\left\|\left\langle x_{n(2)}, x_{i}\right\rangle\right\|<\varepsilon_{2}$ whenever $i>n(3)$ and so on. Set $\varepsilon_{i}=1 / i$. Then we claim the subsequence $\left\{x_{n(i)}\right\}$ of $\left\{x_{k}\right\}$ satisfies 
the condition (10). Indeed

$$
\begin{aligned}
\left\|\frac{1}{k^{2}} \sum_{i, j=1}^{k}\left\langle x_{n(i)}, x_{n(j)}\right\rangle\right\| & \leq \frac{1}{k^{2}}\left(\sum_{i=1}^{k}\left\|x_{n(i)}\right\|^{2}+2 \sum_{1 \leq i<j \leq k}\left\|\left\langle x_{n(i)}, x_{n(j)}\right\rangle\right\|\right) \\
& \leq \frac{1}{k}+\frac{2}{k^{2}}\left((k-1) \varepsilon_{1}+(k-2) \varepsilon_{2}+\cdots+\varepsilon_{k-1}\right) \\
& =\frac{1}{k}+\frac{2}{k^{2}}\left(k+\frac{k}{2}+\frac{k}{3}+\cdots+\frac{k}{k-1}-(k-1)\right) \\
& =\frac{1}{k}+\frac{2}{k^{2}}+\frac{2}{k}\left(\frac{1}{2}+\frac{1}{3}+\cdots+\frac{1}{k-1}\right)= \\
& =\frac{1}{k}+\frac{2}{k^{2}}+\frac{2}{k}(C-1+\ln (k-1)+o(1)),
\end{aligned}
$$

where $C \approx 0,5772$ is the Euler constant and, consequently, the right part of this estimation vanishes when $k$ goes to infinity.

Proposition 5.4. If a Hilbert $C^{*}$-module has the module Schur property, then it has the property (mBS2).

Proof. Let $\left\{x_{k}\right\}$ be a $\tau_{\widehat{V}}$-convergent sequence of a Hilbert $C^{*}$-module $V$ and let $x$ be its limit in the completion of $V$ with respect to the topology $\tau_{\widehat{V}}$. Then Lemma 4.3 ensures $x$ has to belong to $V$ actually. Thus the numerical sequence $\left\{\left\|x_{k}-x\right\|\right\}$ converges to zero. Without loss of generality (because one can pass to a subsequence) we can suppose that $\left\|x_{k}-x\right\| \leq 1 / k^{2}$. This implies

$$
\begin{aligned}
\left\|\frac{1}{n}\left(\sum_{k=1}^{n} x_{k}\right)-x\right\| & =\left\|\frac{1}{n} \sum_{k=1}^{n}\left(x_{k}-x\right)\right\| \\
& \leq \frac{1}{n} \sum_{k=1}^{n}\left\|x_{k}-x\right\| \\
& \leq \frac{1}{n}\left(\sum_{k=1}^{\infty} \frac{1}{k^{2}}\right)
\end{aligned}
$$

and the right part of this expression converges to zero.

Theorem 5.5. A $C^{*}$-algebra with a strictly positive element, in particular, a separable $C^{*}$-algebra, has the property (mBS2) if and only if it is unital, if and only if it has the module Schur property.

Proof. Theorem 4.6 and Proposition 5.4 ensure that a unital $C^{*}$-algebra has the property (mBS2). To check the converse implication let us fix an arbitrary non-unital $C^{*}$-algebra $A$ with a strictly positive element and consider $V=A$ as a Hilbert $A$-module. Then exactly by the same reasons as explained in the proof of Theorem 4.6 any element of the right multiplier algebra $R M(A)$ may be obtained just as a limit of a sequence from $A$ with respect to the right strict topology, which coincides with the topology $\tau_{\widehat{V}}$. Therefore, one can find a sequence $\left\{x_{k}\right\}$ of $A$ which converges with respect to the topology $\tau_{\widehat{V}}$ (for instance, to the unit of $R M(A)$ ), but which does not converge in norm. Then for any 
subsequence $\left\{x_{k(i)}\right\}$ of $\left\{x_{k}\right\}$ the sequence $\left\{\frac{1}{n} \sum_{i=1}^{n} x_{k(i)}\right\}$ of $A$ has obviously the same $\tau_{\widehat{V}^{-}}$ limit in $R M(A)$ as the sequence $\left\{x_{k}\right\}$, so $\left\{\frac{1}{n} \sum_{i=1}^{n} x_{k(i)}\right\}$ cannot converge in norm. Thus $A$ does not have the property (mBS2).

Theorem 5.6. Assume a Hilbert $C^{*}$-module $V$ admits a decomposition into a direct sum $V=V_{1} \oplus V_{2}$. Then the following conditions are equivalent:

(i) $V$ has the property ( $m B S 2)$.

(ii) Both $V_{1}$ and $V_{2}$ have the property (mBS2).

Proof. Obviously, (i) implies (ii) and we have just to ensure the inverse conclusion. Consider any $\tau_{\widehat{V}}$-convergent sequence $\left\{x_{i}\right\}$ of $V$. Then its elements admit decompositions as $x_{i}=x_{i}^{(1)} \oplus x_{i}^{(2)}$ with $x_{i}^{(1)} \in V_{1}, x_{i}^{(2)} \in V_{2}$. Both the sequences $\left\{x_{i}^{(j)}\right\}$ are $\tau_{\widehat{V}_{j}}$-convergent sequences $(j=1,2)$. Therefore, there are subsequences $\left\{x_{i(k)}^{(j)}\right\}$ of $\left\{x_{i}^{(j)}\right\}$ such that their means $\frac{1}{n} \sum_{k=1}^{n} x_{i(k)}^{(j)}$ converge in norm to some elements $x^{(j)}$ of $V_{j}$, respectively for $j=1,2$. Thus, for the sequence $\left\{x_{i(k)}\right\}$ and for $x=x^{(1)} \oplus x^{(2)}$ we have the following estimate:

$$
\begin{aligned}
\left\|\frac{1}{n}\left(\sum_{k=1}^{n} x_{i(k)}\right)-\left(x^{(1)} \oplus x^{(2)}\right)\right\| & =\left\|\left(\frac{1}{n} \sum_{k=1}^{n} x_{i(k)}^{(1)}-x^{(1)}\right) \oplus\left(\frac{1}{n} \sum_{k=1}^{n} x_{i(k)}^{(2)}-x^{(2)}\right)\right\| \\
& \leq\left\|\frac{1}{n} \sum_{k=1}^{n} x_{i(k)}^{(1)}-x^{(1)}\right\|+\left\|\frac{1}{n} \sum_{k=1}^{n} x_{i(k)}^{(2)}-x^{(2)}\right\|
\end{aligned}
$$

Since the upper bounds converge to zero as $k$ tends to infinity, the sum in the first term converges to $x$ in norm. So $V$ has the property (mBS2).

Corollary 5.7. Let $A$ be a $\sigma$-unital $C^{*}$-algebra with the property (mBS2). Then finitely generated Hilbert modules over unital $A$ and finitely generated projective Hilbert modules over A have the property (mBS2).

Let $A$ be a $C^{*}$-algebra and consider the infinite matrix algebra $M_{\infty}(A)=\cup_{n=1}^{\infty} M_{n}(A)$. For vectors $x_{1}, \ldots, x_{n}$ of a Hilbert $A$-module $V$ consider the Gram matrix based on them:

$$
G\left(x_{1}, \ldots, x_{n}\right)=\left(\begin{array}{ccc}
\left\langle x_{1}, x_{1}\right\rangle & \left\langle x_{1}, x_{2}\right\rangle \ldots & \left\langle x_{1}, x_{n}\right\rangle \\
\left\langle x_{2}, x_{1}\right\rangle & \left\langle x_{2}, x_{2}\right\rangle \ldots & \left\langle x_{2}, x_{n}\right\rangle \\
\ldots \ldots \ldots & \ldots \ldots \ldots \ldots & \ldots \ldots \\
\left\langle x_{n}, x_{1}\right\rangle & \left\langle x_{n}, x_{2}\right\rangle \ldots & \left\langle x_{n}, x_{n}\right\rangle
\end{array}\right)
$$

with entries $g_{i j}=\left\langle x_{i}, x_{j}\right\rangle$, besides $G\left(x_{1}, \ldots, x_{n}\right) \in M_{\infty}(A)$. Then the condition (10) on a sequence $\left\{x_{i}\right\}$ of $V$ with $x=0$ can be equivalently reformulated as the assertion that $\left\{\frac{1}{k^{2}} \sum_{i, j=1}^{k} g_{i j}\right\}$ forms a sequence that converges to zero with respect to the norm topology. Instead of condition (10) one can require that the sequence $\left\{\frac{1}{n^{2}} G\left(x_{1}, \ldots, x_{n}\right)\right\}$ of $M_{\infty}(A)$ has to converge to zero in norm. For instance, this condition holds whenever $\left\{x_{i}\right\}$ is an orthogonal system of norm one vectors, because $\left\|G\left(x_{1}, \ldots, x_{n}\right)\right\| \leq \sum_{i, j=1}^{n}\left\|g_{i j}\right\|$ (cf. [21]). Let us remark that in general neither the value $\left\|\frac{1}{n^{2}} \sum_{i, j=1}^{n}\left\langle x_{i}, x_{j}\right\rangle\right\|$ nor the value $\left\|\frac{1}{n^{2}} G\left(x_{1}, \ldots, x_{n}\right)\right\|$ majorize each other. 


\section{Dual modules and module Schur and Banach-Saks Properties}

In this section we establish an interrelation between self-duality of countably generated Hilbert $C^{*}$-modules and their properties to have the module Schur or the module BanachSaks property, respectively. The main goal is an alternative characterization of $C^{*}$-dual Hilbert $C^{*}$-modules of representatives of this class.

Suppose $X$ denotes the completion of $V$ with respect to the topology $\tau_{\widehat{V}}$, and $X_{s}$ denotes the subset of $X$ consisting of all equivalence classes of $\tau_{\widehat{V}}$-Cauchy sequences from $V$. (Two Cauchy sequences are supposed to be equivalent if and only if their difference is a $\tau_{\widehat{V}}$-null sequence.) Let us show how the set $X_{s}$ can be canonically identified with a subset of the dual module $V^{\prime}$, similarly like $V$ is canonically embeddable into its $C^{*}$-dual $A$-module $V^{\prime}$. Let $\left\{x_{i}\right\}$ be a $\tau_{\widehat{V}}$-Cauchy sequence from $V$, i.e. the sequence $\left\{\left\langle x_{i}, z\right\rangle\right\}$ converges in norm to some element $x(z) \in A$ for any $z \in V$ since $\|\langle u, v\rangle\|=\|\langle v, u\rangle\|$ for any $u, v \in V$. As we see this construction defines an $A$-linear functional $\langle x, \cdot\rangle: V \rightarrow A$. Now, for this functional $\langle x, \cdot\rangle$, for $z \in V$ and for any $\varepsilon>0$ there exists a number $i_{0}$ such that

$$
\|x(z)\| \leq\left\|\left\langle x_{i}, z\right\rangle\right\|+\varepsilon \leq\left\|x_{i}\right\|\|z\|+\varepsilon
$$

whenever $i>i_{0}$, where $\left\|x_{i}\right\|<C$ for some constant $C$ and for all $i$ by Proposition 3.3 . Thus, the functional $\langle x, \cdot\rangle$ is bounded and belongs to the dual module $V^{\prime}$.

Proposition 6.1. The topology $\tau_{\widehat{V}}$ has a countable base of neighborhoods of zero provided $V$ is a countably generated Hilbert A-module.

Proof. Let $V=\overline{\operatorname{span}}_{A}\left\{x_{i}: i \in \mathbb{N}\right\}$, i.e. the sequence $\left\{x_{i}\right\}_{i=1}^{\infty}$ generates $V$ over $A$. Then we claim the countable family of sets

$$
\begin{gathered}
U_{x_{i_{1}}, \ldots, x_{i_{n}}, 1 / N}=\left\{y \in V:\left\|\left\langle x_{i_{1}}, y\right\rangle\right\|<1 / N, \ldots,\left\|\left\langle x_{i_{n}}, y\right\rangle\right\|<1 / N\right\} \\
1<i_{1}, \ldots, i_{n}<\infty, n, N \in \mathbb{N}
\end{gathered}
$$

forms the base of neighborhoods of zero for the topology $\tau_{\widehat{V}}$. Indeed, let us take into consideration any $\tau_{\widehat{V}}$-neighborhood of zero of the form

$$
U_{x, \varepsilon}=\{y \in V:\|\langle x, y\rangle\|<\varepsilon\}
$$

where $x \in V, \varepsilon>0$. It is just enough to check that any of these open sets contains one of the sets (11). By supposition, for any $\delta>0$ there are $a_{1}, \ldots, a_{n}$ of $A$ such that

$$
\left\|x-\sum_{i=1}^{n} x_{i} a_{i}\right\|<\delta .
$$

Consider a neighborhood $U_{x_{1}, \ldots, x_{n}, 1 / N}$. By Proposition 3.5 we can assume that the norms of all the elements of the generating set do not exceed one, whenever $N$ is large enough. 
Therefore for any $z \in U_{x_{1}, \ldots, x_{n}, 1 / N}$ one can deduce

$$
\begin{aligned}
\|\langle x, z\rangle\| & \leq\left\|\left\langle x-\sum_{i=1}^{n} x_{i} a_{i}, z\right\rangle\right\|+\left\|\left\langle\sum_{i=1}^{n} x_{i} a_{i}, z\right\rangle\right\| \\
& \leq \delta\|z\|+\sum_{i=1}^{n}\left\|a_{i}\right\|\left\|\left\langle x_{i}, z\right\rangle\right\| \\
& \leq\left(\delta+\frac{1}{N} \sum_{i=1}^{n}\left\|a_{i}\right\|\right)\|z\| .
\end{aligned}
$$

The numerical expression turns out to be less than the selected $\varepsilon$, whenever both $\delta$ and $1 / N$ are small enough and $n \in \mathbb{N}$ is fixed. So we are done.

Corollary 6.2. Let $V$ be a countably generated Hilbert $C^{*}$-module. Then under the notations above $X_{s}$ coincides with the completion $X$ of $V$ with respect to the topology $\tau_{\widehat{V}}$ and, consequently, $X$ can be isometrically embedded into the the $C^{*}$-dual module $V^{\prime}$ extending the canonical embedding of $V$ into $V^{\prime}$.

Theorem 6.3. Let $V$ be a countably generated Hilbert module over a unital $C^{*}$-algebra $A$. Then the completion $X$ of $V$ with respect to the topology $\tau_{\widehat{V}}$ coincides with the dual module $V^{\prime}$ extending the canonical embedding of $V$ into $V^{\prime}$.

Proof. We already know that $X$ is included into $V^{\prime}$ via the canonical embedding of $V$ into $V^{\prime}$ and should just check the coincidence in the particular case. Firstly, let us suppose $V$ is the standard module $l_{2}(A)$. Then we can use the characterization of the dual module $l_{2}(A)^{\prime}$ described in Theorem 2.1. For any $\beta=\left(b_{i}\right) \in l_{2}(A)^{\prime}$, where $\left\|\sum_{i=1}^{n} b_{i}^{*} b_{i}\right\| \leq C$ for all $n$ and some finite constant $C$, let us consider the finite vectors $\alpha_{n}=\left(b_{1}, \ldots, b_{n}, 0,0, \ldots\right) \in l_{2}(A)$. Then for any $y=\left(y_{i}\right) \in l_{2}(A)$ we have

$$
\begin{aligned}
\left\|\left\langle\alpha_{n}-\alpha_{m}, y\right\rangle\right\| & =\left\|\sum_{i=n+1}^{m} b_{i}^{*} y_{i}\right\| \\
& \leq\left\|\sum_{i=n+1}^{m} b_{i}^{*} b_{i}\right\|^{1 / 2}\left\|\sum_{i=n+1}^{m} y_{i}^{*} y_{i}\right\|^{1 / 2} \\
& \leq C^{1 / 2}\left\|\sum_{i=n+1}^{m} y_{i}^{*} y_{i}\right\|^{1 / 2}
\end{aligned}
$$

and this expression goes to zero provided $m, n$ go to infinity. Thus, $\left\{\alpha_{n}\right\}$ is a $\tau_{\widehat{V}^{-}}$-Cauchy sequence and, moreover, its $\tau_{\widehat{V}}$-limit, obviously, coincides with $\beta$. This shows the desirable identification of $X$ and $V^{\prime}$ that extends the canonical embedding of $V$ into $V^{\prime}$.

Now assume $V$ is an arbitrary countably generated Hilbert $A$-module. Then by the Kasparov's stabilization theorem there exists a Hilbert $A$-module $W$ such that $l_{2}(A)=$ $V \oplus W$. Let us denote by $P_{V}: l_{2}(A) \rightarrow l_{2}(A)$ the corresponding orthogonal projection onto the first summand, so Range $\left(P_{V}\right)=V$. Now for any functional $f \in V^{\prime}$ one can define a functional $\tilde{f} \in l_{2}(A)^{\prime}$ as the composition $\tilde{f}=f P_{V}$. Because $l_{2}(A)^{\prime}$ coincides with the $\tau_{l_{2}(A)}$-completion of $l_{2}(A)$ there is a sequence $\left\{z_{i}\right\}$ of $l_{2}(A)$, where $z_{i}=x_{i} \oplus y_{i}$, such 
that its $\tau_{l_{2}(A)}$-limit is $\widetilde{f}$. Thus, the sequence $\left\{x_{i}\right\}$ of $V$ converges to $f$ with respect to the topology $\tau_{\widehat{V}}$.

Remark 6.4. Theorem 6.3 is true also for $\sigma$-unital $C^{*}$-algebras $A$, but the proof involves more complicated techniques and is left to the reader. Actually, it is the corollary of the crucial general result [ $[$, Theorem 6.4] and Proposition [3.3. Let us also stress for $V=A$ that in the non-unital, $\sigma$-unital case the theorem above is equivalent to the well-known fact that the completion of $A$ with respect to the left strict topology coincides with the left multiplier algebra $L M(A)$.

Applying Theorem 6.3 we get:

Corollary 6.5. Let $V$ be a countably generated Hilbert module over a unital $C^{*}$-algebra. Then $V$ is self-dual whenever it has the module Schur property.

Corollary 6.6. Let $V$ be a countably generated self-dual Hilbert module over a unital $C^{*}$-algebra. Then $V$ has the property $(m B S 1)$ if and only if it has the property (mBS2).

Proof. Suppose the property (mBS1) holds for $V$. Consider any $\tau_{\widehat{V}}$-convergent sequence $\left\{x_{i}\right\}$ of $V$. Then by supposition and by Theorem 6.3 its $\tau_{\widehat{V}}$-limit $x$ belongs to $V$. Then the sequence $\left\{y_{i}=x_{i}-x\right\}$ is a $\tau_{\widehat{V}}$-null sequence. Hence, it admits a subsequence $\left\{y_{i(k)}\right\}$ satisfying the equality (9), so the sequence $\frac{1}{n} \sum_{k=1}^{n} x_{i(k)}$ converges to $x$ in norm.

Corollary 6.7. Any countably generated self-dual Hilbert module over a unital $C^{*}$-algebra possesses the property (mBS2).

The proof follows from Theorem 5.3 and Corollary 6.6.

As a final result we can state, that the module Schur and the (mBS2) properties are different in general, because any standard Hilbert module over a finite dimensional $C^{*}$ algebra is self-dual and, consequently, has the property (mBS2), however it does not have the module Schur property by Proposition 4.11,

Acknowledgement: The presented work is part of the research project " $K$-Theory, $C^{*}$ Algebras, and Index Theory" of Deutsche Forschungsgemeinschaft (DFG). The authors are grateful to DFG for the support. The essential part of the research was done during the visit of the second author to the Leipzig University of Applied Sciences (HTWK), and he appreciates its hospitality a lot.

The authors are grateful to A. Ya. Helemskii for helpful discussions.

\section{REFERENCES}

[1] S. BAnACH, S. SAKs, Sur la convergence forte dans les champs $L^{p}$, Studia Math. 2(1930), 51-57.

[2] J. M. F. Castillo, M. GonzÁlez, Three space problems in Banach space theory, Lecture Notes in Mathematics, v. 1667, Springer-Verlag, 1997.

[3] Cho-Ho Chu, The weak Banach-Saks property in $C^{*}$-algebras, J. Funct. Anal. 121(1994), 1-14.

[4] J. Diestel, Geometry of Banach Spaces, Lecture Notes in Math. v. 485, Springer-Verlag, Berlin, 1975.

[5] J. Dixmier, $C^{*}$-Algebras, North-Holland, Amsterdam, 1982.

[6] M. Frank, Self-duality and $C^{*}$-reflexivity of Hilbert $C^{*}$-modules, Zeitschr. Anal. Anwendungen $\mathbf{9}(1990), 165-176$. 
[7] M. Frank, Hilbert $C^{*}$-modules over monotone complete $C^{*}$-algebras, Math. Nachr. 175(1995), 61-83.

[8] M. Frank, Geometrical aspects of Hilbert $C^{*}$-modules, Positivity 3(1999), 215-243.

[9] M. Frank, A. A. Pavlov, Strict essential extensions of $C^{*}$-algebras and Hilbert $C^{*}$-modules (eprint arXiv:math.OA/0710.0586, to appear in J. Operator Theory).

[10] M. Frank, A. A. Pavlov, Banach-Saks properties of $C^{*}$-algebras and Hilbert $C^{*}$-modules Banach J. Math. Anal., 3(2009), 91-102.

[11] M. Hamana, Modules over monotone complete $C^{*}$-algebras, Internat. J. Math. 3(1992), 185-204.

[12] G. G. Kasparov, Hilbert $C^{*}$-modules: The theorems of Stinespring and Voiculescu, J. Operator Theory 4(1980), 133-150.

[13] M. KusudA, $C^{*}$-crossed products of $C^{*}$-algebras with the weak Banach-Saks property, J. Operator Theory 19(2003), 173-183.

[14] M. KusudA, Morita equivalence for $C^{*}$-algebras with the weak Banach-Saks property. II, Proc. Edinburgh Math. Soc. 50(2007), 185-195.

[15] E. C. LANCE, Hilbert $C^{*}$-Modules - a toolkit for operator algebraists, London Mathematical Society Lecture Note Series, v. 210, Cambridge University Press, England, 1995.

[16] H. Lin, Bounded module maps and pure completely positive maps, J. Oper. Theory 26(1991), 121-138.

[17] H. Lin, Injective Hilbert $C^{*}$-modules, Pacific J. Math. 154(1992), 131-164.

[18] V. M. Manuilov, E. V. Troitsky, Hilbert $C^{*}$ - and $W^{*}$-modules and their morphisms, J. Math. Sci. (New York) 98 (2)(2000), 137-201.

[19] V. M. Manuilov, E. V. Troitsky, Hilbert $C^{*}$-Modules, Translations of Mathematical Monographs, vol. 226, American Mathematical Society, Providence, RI, 2005.

[20] A. S. Mishchenko, A. T. Fomenko, The index of elliptic operators over $C^{*}$-algebras, Math. USSR-Izv. 15(1980), 87-112.

[21] G. J. Murphy, $C^{*}$-algebras and Operator Theory, Academic Press, San Diego, 1990.

$[22]$ W. L. Paschke, Inner product modules over $B^{*}$-algebras, Trans. Amer. Math. Soc., 182(1973), 443-468.

[23] W. L. Paschke, The double $B$-dual of an inner product module over a $C^{*}$-algebra $B, C a n$. $J$. Math., 26 (5)(1974), 1272-1280.

[24] A. A. Pavlov, Algebras of multiplicators and spaces of quasimultiplicators, Mosc. Univ. Math. Bull. 53(1998), no. 6, 13-16.

[25] A. A. Pavlov, E. V. Troitsky, Quantization of coverings of compact topological spaces (in preparation).

[26] G. K. Pedersen, $C^{*}$-algebras and Their Automorphism Groups, London Mathematical Society Monographs, Vol. 14, Academic Press Inc., London, 1979.

[27] I. Raeburn, D. P. Williams, Morita Equivalence and Continuous-Trace $C^{*}$-Algebras, Mathematical Surveys and Monographs, Vol. 60, Amer. Math. Soc., Providence, RI, 1998.

[28] N. E. Wegge-Olsen, K-Theory and $C^{*}$-Algebras, Oxford Univ. Press, 1993.

Hochschule für Technik, Wirtschaft und Kultur (HTWK) Leipzig, Fachbereich IMN, PF 301166, D-04251 Leipzig, F.R. Germany

E-mail address: mfrank@imn.htwk-leipzig.de

URL: http://www.imn.htwk-leipzig.de/ mfrank

Dipartimento di Matematica e Informatica, Università degli Studi di Trieste, Piazzale Europa 1, I-34127 Trieste, Italy and All-Russian Institute of Scientific and Technical Information, Russian Academy of Sciences (VINiti RAS), Usievicha Str. 20, 125190 Moscow A-190, Russia

E-mail address: axpavlov@gmail.com

$U R L:$ http://www.axpavlov.com/ 